Research Paper

\title{
Enhancing Transgene Expression from Recombinant AAV8 Vectors in Different Tissues Using Woodchuck Hepatitis Virus Post-Transcriptional Regulatory Element
}

\author{
Lizheng Wang 1, Zixuan Wang 1, Fangfang Zhang 1, Rui Zhu ${ }^{1}$, Jinpeng Bi ${ }^{1}$, Jiaxin Wu ${ }^{1}$, Haihong Zhang ${ }^{1}$, \\ Hui Wu ${ }^{1}$, Wei Kong ${ }^{1,2}$, Bin Yu ${ }^{1 凶}$, Xianghui Yu ${ }^{1,2}{ }^{\circledR}$ \\ 1. National Engineering Laboratory for AIDS Vaccine, School of Life Sciences, Jilin University, Changchun 130012, China \\ 2. Key Laboratory for Molecular Enzymology and Engineering, the Ministry of Education, School of Life Sciences, Jilin University, Changchun 130012, China \\ $\triangle$ Corresponding authors: School of Life Sciences, Jilin University, Changchun 130012, China. Fax: +86 043185167751 . E-mail addresses: yubin@jlu.edu.cn (B. \\ $\mathrm{Yu})$, xianghui@jlu.edu.cn (X. Yu) \\ () Ivyspring International Publisher. Reproduction is permitted for personal, noncommercial use, provided that the article is in whole, unmodified, and properly cited. See \\ http://ivyspring.com/terms for terms and conditions.
}

Received: 2015.10.18; Accepted: 2016.02.04; Published: 2016.04.01

\begin{abstract}
Adeno-associated virus (AAV) vectors have been utilized extensively in gene therapy and gene function studies, as strong transgene expression is a prerequisite for positive outcomes. AAV8 was reported as the most efficient AAV serotype for transduction of the liver, brain and muscle compared with other serotypes. However, AAV8-mediated transduction of human hepatocytes is rather poor with approximately 20-fold lower efficiency compared with that of mouse hepatocytes. Therefore, we applied the woodchuck hepatitis virus post-transcriptional regulatory element (WPRE) to enhance AAV8-mediated transgene expression driven by a combination promoter (CAG promoter) with a CMV-IE enhancer and chicken beta-actin promoter for a more efficient viral vector. Transgene expression from recombinant AAV8 (rAAV8) vectors harboring a red fluorescent protein (RFP) reporter gene with or without WPRE were evaluated in vitro and in vivo. The results demonstrated that WPRE improved AAV8-mediated RFP expression in different cell lines with clear increases of transgene expression in the liver, brain or muscle of animals. The findings of this study will help to substantially reduce the quantity of viral particles that must be injected in order to reach a therapeutic level of transgene expression in gene therapy. Consequently, such dose reductions may lessen the potential risks associated with high doses of viral vectors.
\end{abstract}

Key words: adeno-associated viral vector, WPRE, transgene expression, gene therapy

\section{Introduction}

Recombinant adeno-associated virus (rAAV) vectors have been widely applied in gene therapy due to their advantages demonstrated in preclinical and clinical therapies, including the ability to infect different tissues, long-term foreign gene expression and lack of pathogenicity in animal models [1]. A large number of evolutionarily diverse AAV serotypes have been identified and applied extensively in gene therapy [2, 3]. Although recombinant adeno-associated virus vector serotype 2 (rAAV2) has been most widely used in human trials as an early serotype isolated from humans, vectors of other serotypes have been shown to be more effective in gene delivery with lower immunogenicity in humans and different tissue specificities [4-8]. AAV8 obtained from rhesus monkeys [9] mainly recognizes laminins (LMs) on the cell surface for entering cells, while AAV2 mainly recognizes heparan sulfate proteoglycans (HSPGs). Among the tissues that are susceptible to infection with AAV8 are the liver, brain and muscle [10-12], and this serotype was reported to be the most efficient in transducing these three types of tissues [13-15].

In gene therapies and gene function studies, 
enhanced transgene expression mediated by AAV vectors offers a greater potential for better therapeutic effects. Methods to improve transgene expression from $\mathrm{rAAV}$ vectors include modifying the capsid of rAAV vectors and using expression cassettes (e.g., introns, CMV enhancer, polyadenylation signals) [16]. The woodchuck hepatitis post-transcriptional regulatory element (WPRE) is typically chosen for optimizing gene expression mediated by AAV vectors or other viral vectors as a powerful cis-acting RNA element in the $3^{\prime}$ untranslated region ( $\left.3^{\prime} \mathrm{UTR}\right)$. It functions by modifying RNA polyadenylation and exporting mRNA partly linked to the CRM-1 dependent nuclear export pathway [17, 18]. The positive effect of insertion of the WPRE into the 3' UTR has been widely confirmed in applications to optimize gene expression in a series of vector contexts [19].

In this study, we aimed to construct a more effective rAAV8 vector by using WPRE combined with the CAG promoter, which was previously shown to drive more efficient transgene expression in some cell lines compared with the cytomegalovirus promoter (CMV) [20]. Based on analysis of its effects both in vitro and in vivo, the WPRE was demonstrated to provide a simple means to significantly enhance AAV8-mediated gene expression under the control of the CAG promoter in the liver, muscle and brain of animal models.

\section{Materials and Methods}

\section{Cell lines}

Human emborynic kidney (HEK) 293T cells, mouse myoblast $\mathrm{C} 2 \mathrm{C} 12$ cells, human neuroblastoma SH-SY5Y cells and human hepatocyte Chang liver cells were obtained from the American Type Culture Collection (ATCC; Manassas, VA, USA). Chang liver cells were cultured in RPMI 1640 medium. HEK 293T, C2C12, and SH-SY5Y cells were cultured in DMEM medium.

\section{Preparation of rAAV8 vectors}

Two rAAV8 vectors, AAV8-CAG-RFP and AAV8-CAG-RFP-WPRE, were constructed for this study. The WPRE element used in this study contains nucleotides 1093-1685 (nucleotide numbering scheme of GenBank accession no. J04514). Both constructs contained expression cassettes flanked by AAV2 terminal repeats. Expression of red fluorescent protein (RFP) was driven by the CAG promoter. Details of the constructs are shown in Figure 1A.

The rAAV8 vectors were prepared by using a triple plasmid system, including a plasmid containing the transgene, a plasmid expressing the rep protein and cap protein of AAV8, and a plasmid (containing
E1a, E1b, E2a, VA and E4) acting as a helper for AAV8 packaging. Plasmids were transfected into HEK 293T cells with polyethylenimine (PEI) as the transfection reagent. Cells and medium were harvested together $72 \mathrm{~h}$ post-transfection for purification. The method for purification of rAAV8 vectors was reported previously [21]. Purified rAAV8 vectors were obtained after three steps: chloroform treatment, PEG8000/ NaCl precipitation and chloroform extraction. Real-time quantitative PCR analysis was used to determine the particle genome titer expressed as viral genomes (vg).

\section{Viral infection}

HEK 293T cells, C2C12 cells, SH-SY5Y cells and Chang liver cells were separately cultured in medium with $10 \%$ fetal bovine serum (FBS) to $70-80 \%$ confluency. The rAAV8 vectors were added to the culture after changing the medium to that containing $2 \%$ FBS. RFP expression was detected by fluorescence microscopy after 3 or 4 days. Image-Pro Plus 6.0 software was used to determine the mean fluorescence intensity (MFI) from images, and the relative MFI was calculated by the data measured. $P$ values were analyzed by t-test.

\section{rAAV8-mediated gene transduction in vivo}

In total 16 male Balb/c mice (20-23 g) and 6 male Wistar rats (180-220 g) were used in this study. The experimental protocol was approved by the University Committee on the Use and Care of Animals of Jilin University of China.

Rats were chosen to study the effect of WPRE in the brain, as microinjections into the striatal regions can be easily performed in this animal model. Rats were placed in a stereotaxic apparatus after being anesthetized with pentobarbital sodium and then received rAAV8 $\left(3 \times 10^{9} \mathrm{vg}\right.$ viral vectors in $3 \mu \mathrm{L}$ PBS $)$ in the right striatum (coordinates from the bregma: $\mathrm{AP}+0.6 \mathrm{~mm}, \mathrm{ML}-0.2 \mathrm{~mm}, \mathrm{DV}-5 \mathrm{~mm})$ with a Hamilton syringe ( $0.46 \mathrm{~mm}$ in diameter, blunt tip) at a rate of $0.5 \mu \mathrm{L}$ per min. The needle was left in place for $8 \mathrm{~min}$ and then slowly withdrawn in the subsequent 3 to 4 min.

rAAV8 vectors were injected directly into the muscle of mice at the dose of $1.5 \times 10^{11} \mathrm{vg}$ in $50 \mu \mathrm{L}$ PBS. The livers of mice were exposed after being anesthetized for the subsequent injection of rAAV vectors at the dose of $1.5 \times 10^{11} \mathrm{vg}$ in $50 \mu \mathrm{L}$ PBS.

\section{Biophotonic imaging}

Three weeks after administration of the AAV8-CAG-RFP or AAV8-CAG-RFP-WPRE vector, animals were killed to isolate target organs, which were placed in cold PBS immediately. Within $15 \mathrm{~min}$, images were obtained by using an In Vivo Imaging 
System Fx Pro (Kodak, USA). The excitation wavelength (lambda Ex) was $530 \mathrm{~nm}$, and the emission wavelength (Em) was $600 \mathrm{~nm}$ for RFP imaging.

\section{Quantitative real-time PCR (qRT-PCR) for detection of RFP mRNA}

Parts of tissues with RFP were cut immediately following bio-fluorescence imaging to extract total RNA using an RNeasy kit (Qiagen, Valenca, CA, USA). RFP mRNA was analyzed via qRT-PCR after cDNA synthesis by using the PrimeScript 1st Strand cDNA Synthesis Kit (Takara Biotechnology Co., Ltd, Dalian,China). The relative mRNA level was calculated, and $P$ values were analyzed using T-test.

\section{Results}

\section{Generation and characterization of rAAV8 vectors}

Viral vectors were packaged in HEK 293T cells. After purification, we harvested two rAAV8 vectors. The viral titers were detected by quantitative RT-PCR with a pair of primers (F: AGACGCAGCCAC AAAGATCC; R: CACTCTCATCTTCCGCAGGT) amplifying part of the CAG promoter sequence. The titers were $3 \times 10^{12} \mathrm{vg} / \mathrm{mL}$ for AAV8-CAG-RFP and $4.7 \times 10^{12} \mathrm{vg} / \mathrm{mL}$ for AAV8-CAG-RFP-WPRE. These viral vectors were validated by PCR to contain the
RFP or RFP and WPRE gene segments as shown in Figure 1B. The RFP gene expression was quantified by RT-PCR in HEK 293T cells transduced with these two viral vectors. The results showed that the RFP gene expressed in AAV8-RFP-transduced cells was 104 $\pm 1.6 \%$ of that in AAV8-RFP-WPRE-transduced cells, although these values were not significantly different. Thus, the two vectors were considered to have nearly the same infection efficiency.

\section{WPRE enhances RFP expression from rAAV8 vectors in several cell lines}

To characterize the effect of the WPRE on CAG promoter activity, we compared RFP expression in HEK 293T cells, SH-SY5Y human neuroblastoma cells, human hepatocyte Chang liver cells and mouse myoblast C2C12 cells. Cells were cultured in 24-well plates and incubated with viral vectors at $9 \times 10^{10} \mathrm{vg}$ per well. RFP expression in C2C12 cells was observed at $72 \mathrm{~h}$ post-transduction, while it was detected at $48 \mathrm{~h}$ post-transduction in the other three cell types. The expression of RFP was clearly improved by the presence of the WPRE in each cell line tested (Figure 2A). The MFI in each image was measured with Image-Pro Plus software, and the results showed that the AAV8-CAG-WPRE vector produced a 2 3-fold greater increase in RFP expression compared with AAV8-CAG-RFP (Figure 2B).

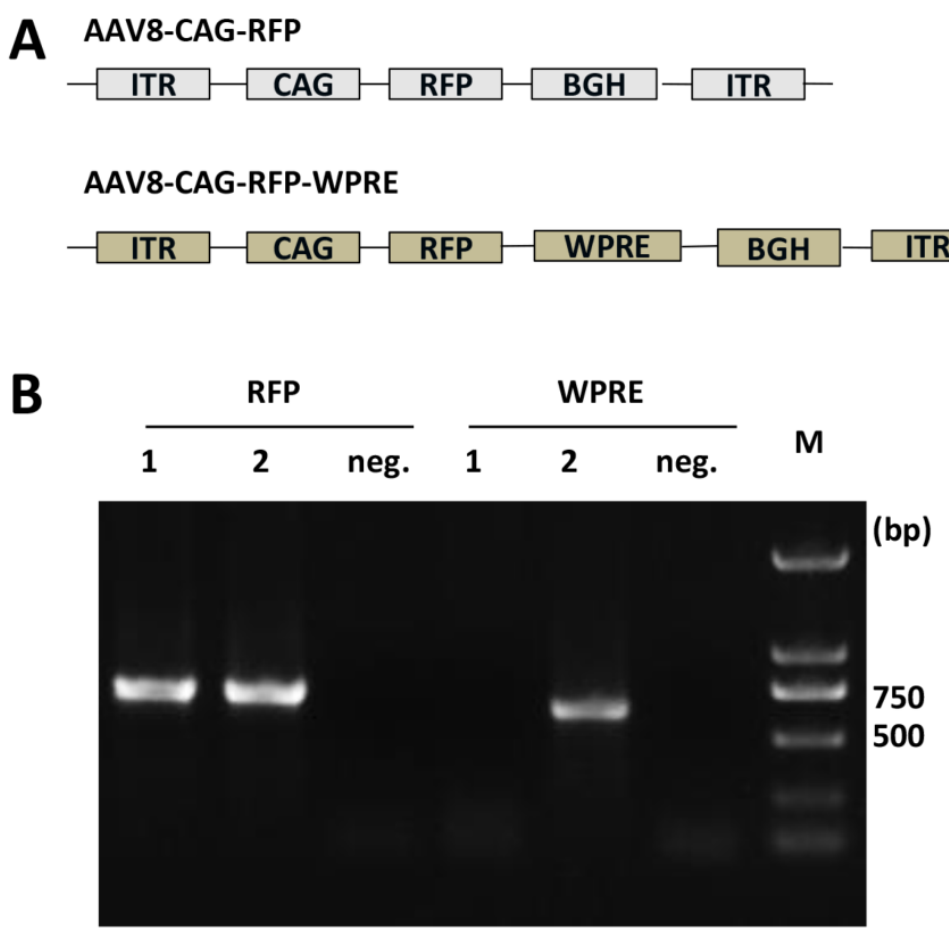

Figure 1. (A) Schematic representation of viral vectors. ITR, inverted terminal repeat; CAG, combination promoter with CMV-IE enhancer and chicken beta-actin promoter; RFP, red fluorescent protein; BGH, bovine growth hormone derived polyadenylation site; WPRE, Woodchuck hepatitis virus post-transcriptional regulatory element. (B) Identification of AAV8 vectors purified by PCR. Gene elements of RFP and WPRE were amplified by PCR from purified viral templates. 1, AAV8-CAG-RFP vectors; 2, AAV8-CAG-RFP-WPRE vectors; neg, negative controls without template. 
A

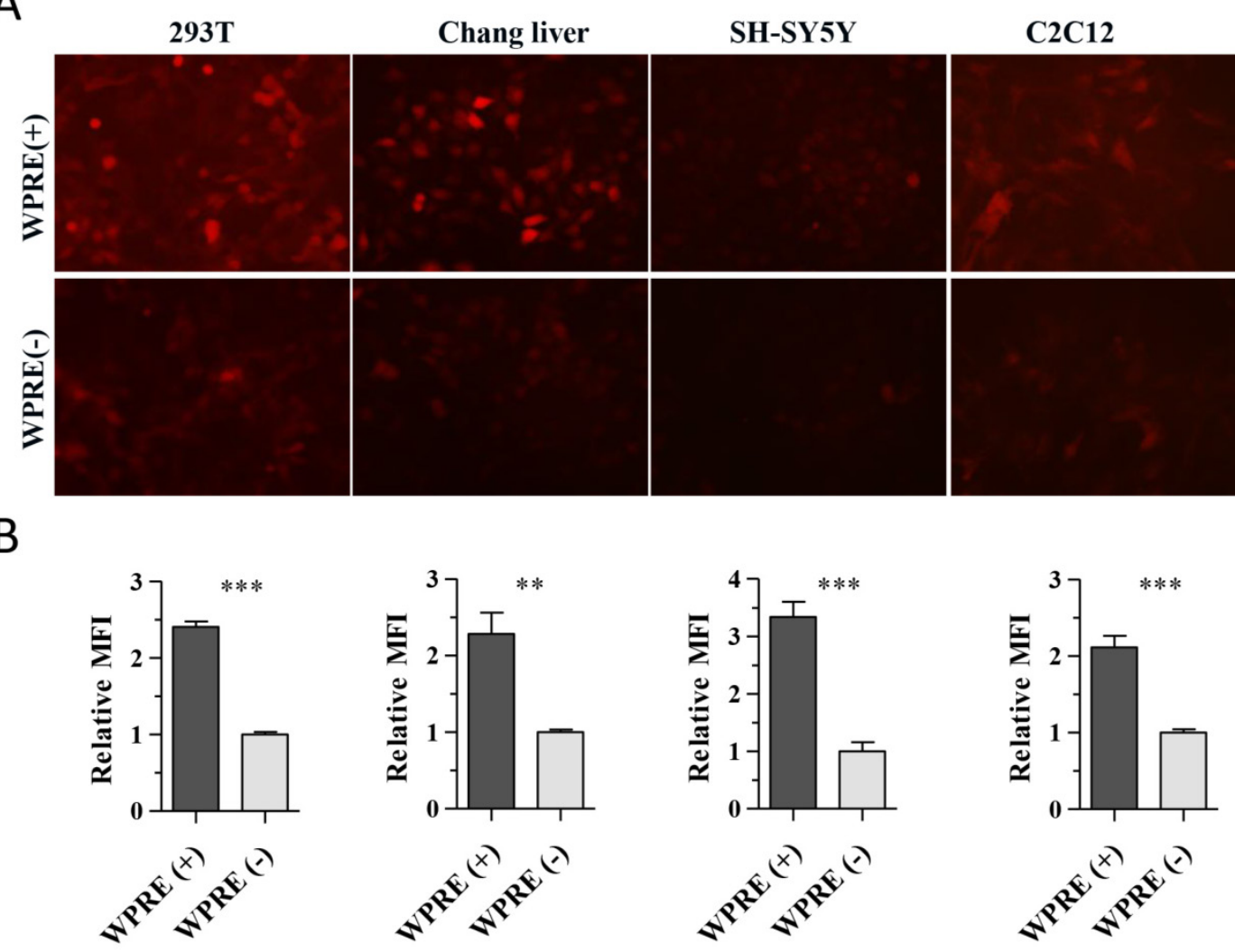

Figure 2. Enhancement of RFP expression by WPRE in vitro. (A) HEK 293T, Chang liver, SH-SY5Y and C2C12 cells were transduced with the rAAV8 vectors at a dose of $9 \times 10^{10} \mathrm{vg}$ per well of a 24-well plate. RFP expression was observed under a fluorescent microscope. (B) Relative MFI was calculated from the absolute MFI measured by the Image-Pro Plus 6.0 software (p-values: $*<0.05, * *<0.005, * * *<0.001)$.

\section{WPRE improves transgene expression in vivo}

The effect of the addition of WPRE on the CAG promoter in AAV8 vectors was evaluated in the liver, brain and muscle in animals. Two weeks after administration of rAAV8 vectors, animals were killed. The brain of rats and the muscle or liver of mice were separated to detect the expression of RFP. Differences of RFP expression could be distinguished easily in all tissue types (Figure 3).

To verify the effect of the WPRE further, RFP mRNA expression levels in different tissues were detected by qRT-PCR. At least three animals were analyzed from the AAV8-CAG-RFP group or AAV8-CAG-RFP-WPRE group. As shown in Figure 4, WPRE showed an approximately 5-fold enhancement of RFP mRNA expression in the mouse liver, while the increase was $\sim 4$-fold in the rat brain and $\sim 2$ in the mouse muscle compared with controls without WPRE.

\section{Discussion}

High efficiency of transgene expression is a fundamental requirement in gene therapy and gene function research. Many methods have been evaluated to achieve a higher level of transgene product in target cells. WPRE, which is able to improve gene expression at a post-transcriptional level, has been used to successfully enhance transgene expression in the optimization of viral vectors, such as adenovirus, lentiviral and AAV vectors [22-24].

Although WPRE has been applied in AAV vectors, these studies were mostly based on AAV2 $[25,26]$. AAV8 may have a greater potential for use in applications in which the target is the liver, brain or muscle, as it was reported to have a stronger ability to infect these and other tissues compared with other AAV serotypes [13-15]. rAAV8 has been used to treat hemophilia based on its hepatotropism and achieved a more successful therapeutic effect compared with rAAV2 [27]. rAAV8 vectors were also applied in gene therapy for Duchenne's muscular dystrophy (DMD), and the mice that received treatment gained improved muscle strength as determined by behavioral and histopathological evaluations [28]. Meanwhile, rAAV8 vectors also have been utilized extensively in the nervous system targeting primarily neurons [29]. Despite the effectiveness of rAAV8 vectors in many animal models, their transduction levels of human hepatocytes were found to be rather poor with approximately 20 times lower efficiency compared with that of mouse hepatocytes [30, 31]. 
This difference indicates that further optimization of rAAV8 vectors would be valuable. In this study, we designed a rAAV8 vector with the additional WPRE under the control of the CAG promoter to achieve stronger transgene expression.

WPRE-mediated enhancement of transgene expression was reported to be promoter and cell specific [32]. The effect of WPRE on retroviral vectors was different when combined with the heterologous mouse mammary tumor virus (MMTV) promoter or whey acidic protein (WAP) promoter. When we tested the effect of WPRE on the CAG promoter in the AAV8 vector in different cell lines or tissues, the results revealed a positive enhancement in all four cell lines (Figure 2). Ramezani et al. tested WPRE in combination with a series of promoters in lentiviral vectors, and this element showed a limited effect in enhancing transgene expression under the control of the CAG promoter in 293T cells [33]. By contrast, WPRE served to increase AAV8 transduction in 293T cells as well as other cell types in the present study. As shown in Figures 3 and 4, WPRE clearly enhanced transgene expression at both the protein and mRNA levels. Since most published reports on the enhancement of transgene expression by WPRE have been limited to in vitro experiments or certain tissues in animals, the demonstration of WPRE-mediated enhancement in various tissues infected by AAV8 in this study may widen the range of applications of AAV8 vectors containing this element in gene therapy or gene function research [22, 24, 34].

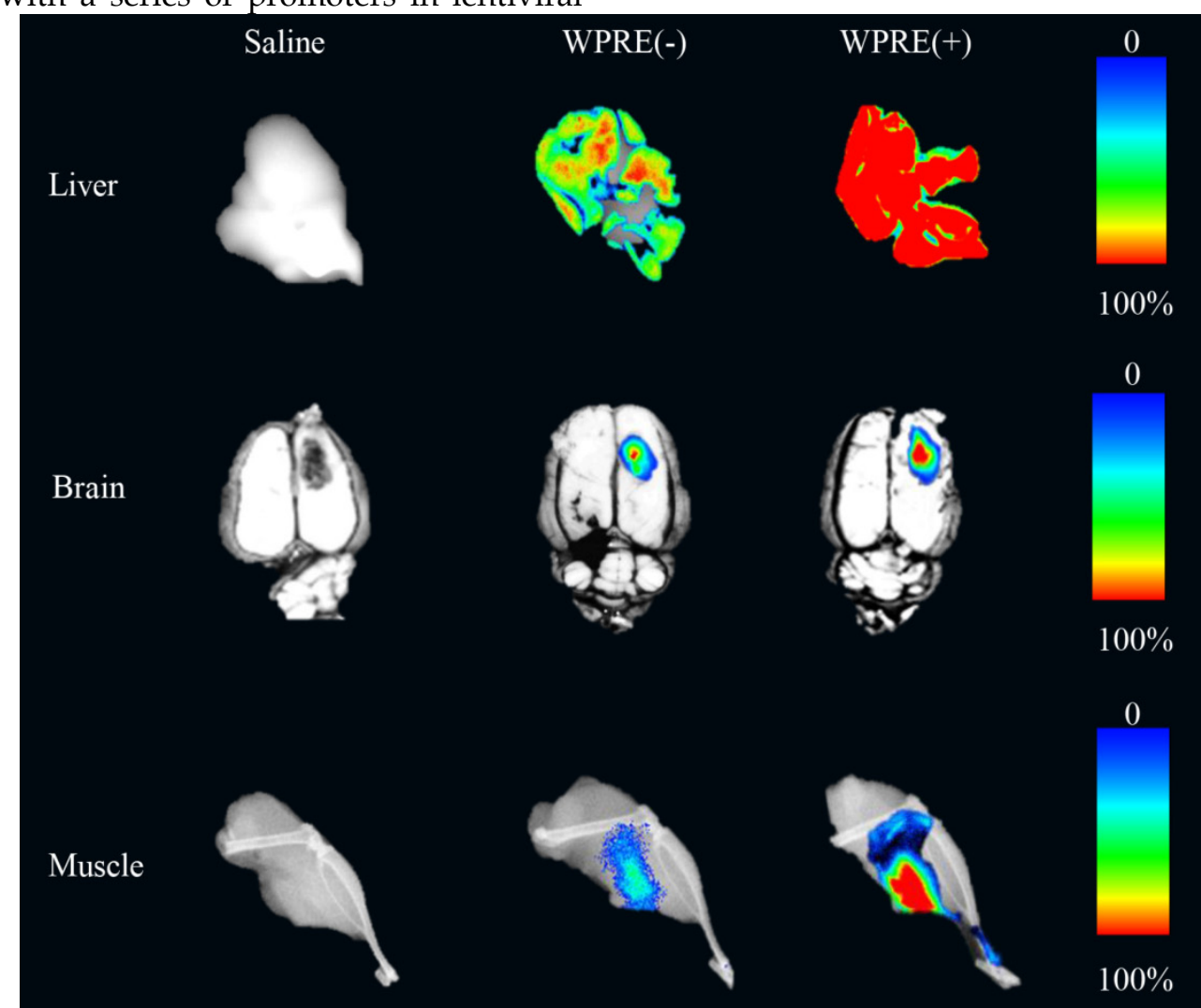

Figure 3. Biophotonic imaging of RFP in different tissues. AAV8-CAG-RFP [WPRE(-)] and AAV8-RFP-WPRE vectors [WPRE(+)] were injected directly into the liver or muscle of mice, while they were administered in the striatum of the brain in rats. Expression of RFP in tissues injected with saline, standard viruses or viruses containing WPRE is shown. Tissues receiving saline were used as negative controls.
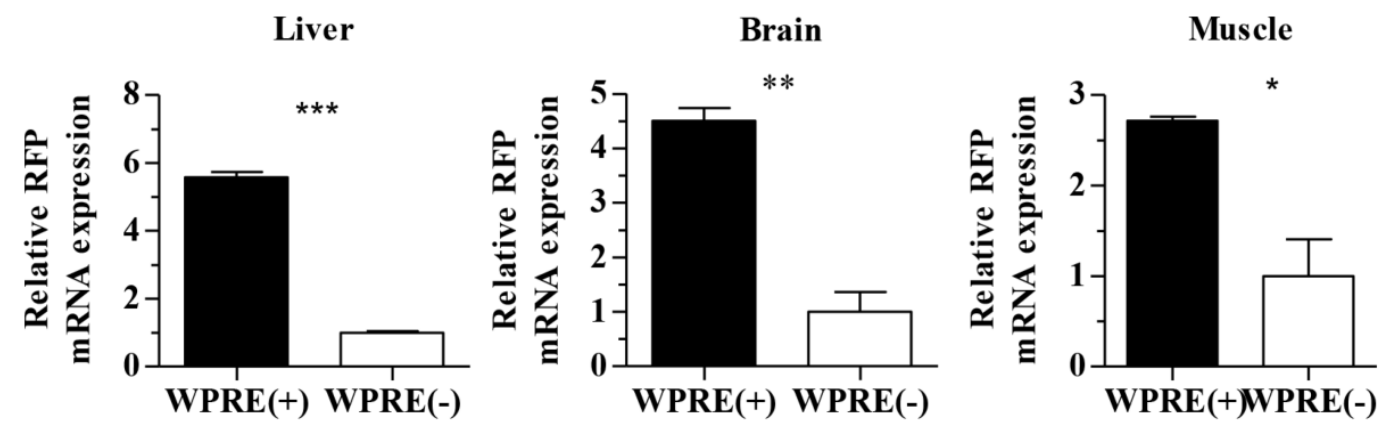

Figure 4. Real-time RT-PCR analysis of RFP mRNA levels in tissues transduced with viral vectors. Relative increases in RFP mRNA levels of AAV8-CAG-RFP and AAV8-CAG-RFP-WPRE vectors are shown (p-values: $*<0.05, * *<0.005, * * *<0.001$ ). 
In summary, WPRE was shown here to increase the transgene expression from AAV8 vectors under the control of the CAG promoter in vitro and in vivo. The design of a more effective rAAV8 vector with WPRE will substantially reduce the quantity of viral particles that must be injected in order to reach a therapeutic level of transgene expression.

\section{Acknowledgements}

This study was supported by funding from the National Nature Science Foundation of China (nos. 31300756 and 81472816) and the Key Projects in the National Science \& Technology Pillar Program in the Twelfth Five-year Plan Period (Grant no. 2013ZX10004608-003). We also wish to acknowledge Phuong Thi Sarkis for editorial support in the preparation of this manuscript.

\section{Competing Interests}

The authors have declared that no competing interest exists.

\section{References}

1. Mueller C, Flotte TR. Clinical gene therapy using recombinant adeno-associated virus vectors. Gene therapy. 2008; 15: 858-63.

2. Schultz BR, Chamberlain JS. Recombinant adeno-associated virus transduction and integration. Molecular therapy : the journal of the American Society of Gene Therapy. 2008; 16: 1189-99.

3. Gao G, Vandenberghe LH, Wilson JM. New recombinant serotypes of AAV vectors. Curr Gene Ther. 2005; 5: 285-97.

4. Arimura S, Okada T, Tezuka T, Chiyo T, Kasahara Y, Yoshimura T, et al. Neuromuscular disease. DOK7 gene therapy benefits mouse models of diseases characterized by defects in the neuromuscular junction. Science. 2014; 345: 1505-8.

5. Burger C, Gorbatyuk OS, Velardo MJ, Peden CS, Williams P, Zolotukhin S, et al. Recombinant AAV viral vectors pseudotyped with viral capsids from serotypes 1, 2, and 5 display differential efficiency and cell tropism after delivery to different regions of the central nervous system. Molecular therapy : the journal of the American Society of Gene Therapy. 2004; 10: 302-17.

6. Mingozzi F, High KA. Therapeutic in vivo gene transfer for genetic disease using AAV: progress and challenges. Nature reviews Genetics. 2011; 12: 341-55.

7. Zincarelli C, Soltys S, Rengo G, Rabinowitz JE. Analysis of AAV serotypes 1-9 mediated gene expression and tropism in mice after systemic injection. Molecular therapy : the journal of the American Society of Gene Therapy. 2008; 16: 1073-80.

8. Boutin S, Monteilhet V, Veron P, Leborgne C, Benveniste O, Montus MF, et al. Prevalence of serum IgG and neutralizing factors against adeno-associated virus (AAV) types $1,2,5,6,8$, and 9 in the healthy population: implications for gene therapy using AAV vectors. Human gene therapy. 2010; 21: 704-12.

9. Gao GP, Alvira MR, Wang LL, Calcedo R, Johnston J, Wilson JM. Novel adeno-associated viruses from rhesus monkeys as vectors for human gene therapy. Proceedings of the National Academy of Sciences of the United States of America. 2002; 99: 11854-9.

10. Nam HJ, Gurda BL, McKenna R, Potter M, Byrne B, Salganik M, et al. Structural Studies of Adeno-Associated Virus Serotype 8 Capsid Transitions Associated with Endosomal Trafficking. J Virol. 2011; 85: 11791-9.

11. Daya S, Berns KI. Gene Therapy Using Adeno-Associated Virus Vectors. Clin Microbiol Rev. 2008; 21: 583-93.

12. Akache B, Grimm D, Pandey K, Yant SR, Xu H, Kay MA. The 37/67-kilodalton laminin receptor is a receptor for adeno-associated virus serotypes $8,2,3$, and 9. J Virol. 2006; 80: 9831-6.

13. Nakai H, Fuess S, Storm TA, Muramatsu S, Nara Y, Kay MA. Unrestricted hepatocyte transduction with adeno-associated virus serotype 8 vectors in mice. J Virol. 2005; 79: 214-24

14. Wang Z, Zhu T, Qiao CP, Zhou LQ, Wang B, Zhang J, et al. Adeno-associated virus serotype 8 efficiently delivers genes to muscle and heart. Nat Biotechnol. 2005; 23: 321-8.

15. Klein RL, Dayton RD, Leidenheimer NJ, Jansen K, Golde TE, Zweig RM. Efficient neuronal gene transfer with AAV8 leads to neurotoxic levels of tau or green fluorescent proteins. Molecular therapy : the journal of the American Society of Gene Therapy. 2006; 13: 517-27.
16. Powell SK, Rivera-Soto R, Gray SJ. Viral expression cassette elements to enhance transgene target specificity and expression in gene therapy. Discovery medicine. 2015; 19: 49-57.

17. Popa I, Harris ME, Donello JE, Hope TJ. CRM1-dependent function of a cis-acting RNA export element. Molecular and cellular biology. 2002; 22: 2057-67.

18. Donello JE, Loeb JE, Hope TJ. Woodchuck hepatitis virus contains a tripartite posttranscriptional regulatory element. J Virol. 1998; 72: 5085-92.

19. Schwenter F, Deglon N, Aebischer P. Optimization of human erythropoietin secretion from MLV-infected human primary fibroblasts used for encapsulated cell therapy. J Gene Med. 2003; 5: 246-57.

20. Shoji I, Aizaki H, Tani H, Ishii K, Chiba T, Saito I, et al. Efficient gene transfer into various mammalian cells, including non-hepatic cells, by baculovirus vectors. The Journal of general virology. 1997; 78 ( Pt 10): 2657-64.

21. Wu XB, Dong XY, Wu ZJ, Cao H, Niu DB, Qu JG, et al. A novel method for purification of recombinant adeno-associated virus vectors on a large scale. Chin Sci Bull. 2001; 46: 485-9.

22. Ge J, Jin L, Tang X, Gao D, An Q, Ping W. Optimization of eGFP expression using a modified baculovirus expression system. Journal of biotechnology. 2014; 173: 41-6.

23. Zhang Y, Gao Y, Speth RC, Jiang N, Mao Y, Sumners C, et al. Adenoviral and adeno-associated viral vectors-mediated neuronal gene transfer to cardiovascular control regions of the rat brain. International journal of medical sciences. 2013; 10: 607-16.

24. Mao Y, Yan R, Li A, Zhang Y, Li J, Du H, et al. Lentiviral Vectors Mediate Long-Term and High Efficiency Transgene Expression in HEK 293T cells. International journal of medical sciences. 2015; 12: 407-15.

25. Decressac M, Mattsson B, Lundblad M, Weikop P, Bjorklund A. Progressive neurodegenerative and behavioural changes induced by AAV-mediated overexpression of alpha-synuclein in midbrain dopamine neurons. Neurobiology of disease. 2012; 45: 939-53.

26. Loeb JE, Cordier WS, Harris ME, Weitzman MD, Hope TJ. Enhanced expression of transgenes from adeno-associated virus vectors with the woodchuck hepatitis virus posttranscriptional regulatory element: Implications for gene therapy. Human gene therapy. 1999; 10: 2295-305.

27. Sarkar R, Tetreault R, Gao GP, Wang LL, Bell P, Chandler R, et al. Total correction of hemophilia A mice with canine FVIII using an AAV 8 serotype. Blood. 2004; 103: 1253-60.

28. Qiao CP, Li JB, Jiang JG, Zhu XD, Wang B, Li J, et al. Myostatin propeptide gene delivery by adeno-associated virus serotype 8 vectors enhances muscle growth and ameliorates dystrophic phenotypes in $\mathrm{mdx}$ mice. Human gene therapy. 2008; 19: 241-54B.

29. Detrait ER, Bowers WJ, Halterman MW, Giuliano RE, Bennice L, Federoff HJ, et al. Reporter gene transfer induces apoptosis in primary cortical neurons. Molecular Therapy. 2002; 5: 723-30.

30. Nietupski JB, Hurlbut GD, Ziegler RJ, Chu Q, Hodges BL, Ashe KM, et al. Systemic administration of AAV8-alpha-galactosidase A induces humoral tolerance in nonhuman primates despite low hepatic expression. Molecular therapy : the journal of the American Society of Gene Therapy. 2011; 19: 1999-2011.

31. Manno CS, Pierce GF, Arruda VR, Glader B, Ragni M, Rasko JJ, et al. Successful transduction of liver in hemophilia by AAV-Factor IX and limitations imposed by the host immune response. Nature medicine. 2006; 12: 342-7.

32. Klein R, Ruttkowski B, Knapp E, Salmons B, Gunzburg WH, Hohenadl C. WPRE-mediated enhancement of gene expression is promoter and cell line specific. Gene. 2006; 372: 153-61.

33. Ramezani A, Hawley TS, Hawley RG. Lentiviral vectors for enhanced gene expression in human hematopoietic cells. Molecular therapy : the journal of the American Society of Gene Therapy. 2000; 2: 458-69.

34. Virella-Lowell I, Zusman B, Foust K, Loiler S, Conlon T, Song S, et al. Enhancing rAAV vector expression in the lung. J Gene Med. 2005; 7: 842-50. 